\title{
NiTi porous structure with 3D interconnected microchannels using steel wire spaceholders
}

\author{
C. Bewerse ${ }^{1}$, A.A. Emery ${ }^{1,3}$, L.C. Brinson ${ }^{1,2}$, D.C. Dunand ${ }^{1 *}$ \\ ${ }^{1}$ Department of Materials Science and Engineering, Northwestern University, \\ Evanston, IL, 60208, USA. \\ ${ }^{2}$ Department of Mechanical Engineering, Northwestern University, \\ Evanston, IL, 60208, USA. \\ ${ }^{3}$ Swiss Federal Institute of Technology Lausanne (EPFL), Lausanne Switzerland
}

\section{Corresponding Author (*):}

David C. Dunand

Email: dunand@northwestern.edu

Tel: $1 / 847 / 491-5370$

Department of Materials Science and Engineering

Northwestern University

2220 Campus Drive, Cook Hall 2036

Evanston, IL 60208

Keywords: NiTi, powder metallurgy, spaceholder, hot isostatic pressing, porous structure

\begin{abstract}
NiTi porous structures with fully 3D interconnected microchannels were created by a powder-metallurgy method using steel wires as spaceholders. Prealloyed NiTi powders were near-fully densified by hot pressing within a high carbon steel wire scaffold, which was then electrochemically dissolved. This resulted in a regular 3D network of orthogonally interconnected microchannels with ellipsoidal cross-sections with $60 \%$ volume fraction. The
\end{abstract}


measured elastic stiffness of $14 \mathrm{GPa}$ compares well to porous and composite models, as well as finite element modeling despite varying geometry and deformation model assumptions. The structure, which is martensitic at room temperature, exhibits brittle fracture at a relatively low stress of $88 \mathrm{MPa}$ due to a TiC interphase at all NiTi powder boundaries. The volume fraction, orientation, shape, and spatial distribution of the microchannels is fully controlled with this method. This makes the structure attractive for biomedical applications, specifically bone implants. The potential shape memory properties achievable through optimized processing would also make the structure effective for energy absorption or actuators.

\section{Introduction}

Nickel Titanium is of interest in several industries due to its ability to recovery strain through the shape memory or superelastic effects. This phenomenon also results in high toughness and excellent mechanical strength, making the material attractive for actuation and energy absorption application [1,2]. Making the material into a porous structure further enhances these attributes by increasing heat transfer capability and the geometric energy absorption advantages. As a biocompatible [3] porous structure, NiTi is also applicable to biomedical applications. The stiffness of the material can be lowered through porosity to match that of bone, while allowing bone cell ingrowth to permanently attach the structure. Many methods have been investigated to create NiTi porous structures, with a variety of pore size, shape, density, and orientations. Surface and bulk porosity techniques have been reviewed by [4]

for orthopedic applications, while general NiTi implants have been reviewed by [5]. Many porous processing techniques for NiTi have also been reviewed in [3], which focuses on the biocompatibility of these structures. 
Powder metallurgy methods with spaceholders have been used to create equiaxed porosity using a variety of different spaceholder materials. Salts such as $\mathrm{NaF}$ [6] and $\mathrm{NaCl}$ [7] have been used as a spaceholder during NiTi hot isostatic pressing after which the continuous salt phase are removed by dissolution in water. $\mathrm{NaCl}$ has also been used as a spaceholder with liquid phase sintering, where the salt is evaporated and the NiTi powders are bonded by small amounts of a NiTi-Nb liquid eutectic phase [8]. The resulting porous structures have high open porosity and interconnectivity, with equiaxed pores. Magnesium was also utilized as a spaceholder during sintering of NiTi powders to create similar networks of equiaxed pores with very high porosity (upwards of 80\%) [9]. Equiaxed porosity has also been created using creep expansion of argon-filled pores, but these pores have no interconnectivity [10]. While these methods cover a wide range of porosity, they provide little control over the spatial distribution of the pores or the interconnectivity between them.

Techniques have also been developed to create elongated porosity structures. In Self Propagating High Temperature Synthesis (SHS), a compacted pellet of Ni and Ti elemental powders is ignited, forming a foam with wavy, interconnected, elongated pores [11]. However this technique also forms undesirable intermetallic phases, which often embrittle the structure [12]. Continuous zone melting can also create elongated porosity in NiTi structures [13], but these pores are closed, with no interconnectivity. The spaceholder method has also been used with steel meshes during NiTi powder HIPing to form, after electrochemical steel removal, 2D interconnected porous NiTi structures of wavy microchannels [14]. Liquid phase sintering of NiTi powders in the presence of scaffolds of steel tubes achieved a microchannel network connected in 3D [15], though difficulties in scalability prevented full-scale analysis of the structure. 
Here, we present a method using powder metallurgy with high carbon steel wire spaceholders to create a fully interconnected 3D porous network of orthogonally stacked, straight microchannels. The spatial distribution, size, shape, and volume fraction are fully tailorable though this method, and the scalability is limited only by load capacity of hot pressing equipment. Microchannels along two dimensions are created directly by the steel wire spaceholders, while interconnectivity of the pore network in the third dimension is created by the intersection of these orthogonal channels.

\section{Experimental Methods}

To create NiTi porous structures with regular arrays of micro-channels, pre-alloyed NiTi powders (Special Metals Corp. Inc.) with a nominal composition of 51.4 at\% Ti, sieved to 44-63 $\mu \mathrm{m}$ were densified in the presence of high-carbon steel music wire (ASTM A228, 0.70-1.00 wt.\% C, $0.20-0.70$ wt. $\%$ Mn, 0.10-0.30 wt.\% Si ) with a diameter of $400 \mu \mathrm{m}$. The $19 \mathrm{~mm}$ long wires were arranged parallel to each other in layers with a center to center spacing of $1 \mathrm{~mm} \pm 100 \mu \mathrm{m}$, using steel inserts with $500 \mu \mathrm{m}$ width grooves spaced at $500 \mu \mathrm{m}$ intervals. The scaffold was created by stacking a total of 24 layers into a $0 / 90^{\circ}$ lay-up, resulting in $1 \mathrm{~cm}^{3}$ of fully orthogonally stacked wires, as shown in Figure 1. 

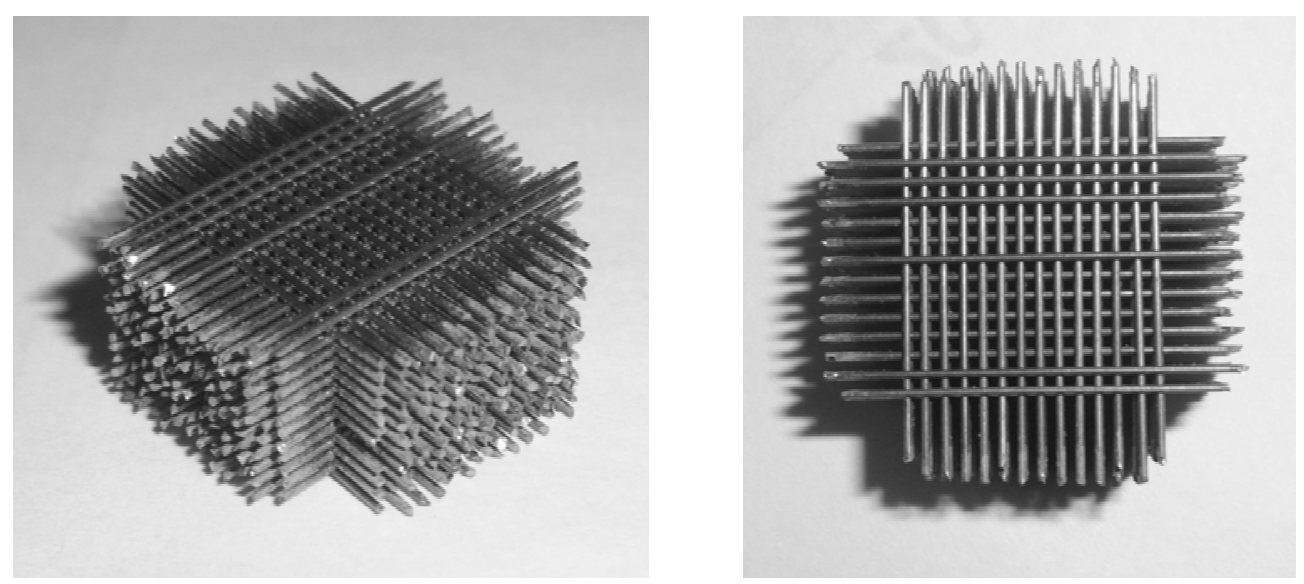

Figure 1: Assembled and sintered spaceholder scaffold constructed with orthogonally stacked high-carbon steel wires with a diameter of $400 \mu \mathrm{m}$.

The wires in the loose, assembled scaffold were sintered together at $960^{\circ} \mathrm{C}$ for $4 \mathrm{hr}$ under a $10^{-5}$ torr vacuum. The empty space within the scaffold was then filled with NiTi powders by tap-packing in a $25.4 \mathrm{~mm}$ inner diameter TZM die coated with boron nitride. The powders were wet by water within the die to remove air pockets and completely fill the steel scaffold, where 34 vol.\% of the packed composite is comprised of the steel scaffold. The wire/powder preform was densified by die hot pressing parallel to the wire stacking direction for $6 \mathrm{hr}$ at $40 \mathrm{MPa}$ and $1020^{\circ} \mathrm{C}$ under a $10^{-5}$ torr vacuum, with the hot-pressing direction perpendicular to the stacked wires. The densified composite was Electrical Discharge Machined (EDM) into $6 \times 6 \times 12 \mathrm{~mm}$ samples, with the long axis machined parallel to the densification direction, and short axis parallel to the wires.

The steel wires were removed from the densified samples using electrochemical dissolution as described in [14], where the NiTi is galvanically protected and the steel is selectively dissolved. Commercial purity $\mathrm{Ti}$ sheet with $0.5 \mathrm{~mm}$ thickness was used for electrodes in an electrolyte bath of supersaturated $\mathrm{NaCl}$ with $3 \%$ acetic acid in deionized water. The NiTi- 
steel composite sample was attached to the Ti anode with nylon wire, and centered within the $\mathrm{Ti}$ sheet cathode wrapped around the inside of a $75 \mathrm{~mm}$ diameter glass beaker. A $0.8 \mathrm{~V}$ voltage bias was used to dissolve the steel while ultra-sonication helped remove the dissolution product and refresh the electrolyte at the surface of the dissolving wires within the growing channels. The current was monitored throughout dissolution. The mass of the sample was periodically measured and full removal of the steel was determined by stabilization of the mass. A sample with dimensions of $6 \times 6 \times 12 \mathrm{~mm}$ reached full dissolution of the steel wires in 180 hours. This dissolution time is very long as compared to [14,17], in which 6 x 6 x 12 mm samples with lowcarbon steel mesh spaceholders of comparable NiTi:steel volume fraction were dissolved in 2535 hours or to [15], where stainless steel tubes, also with a comparable volume fraction, were dissolved in $\sim 10-12$ hours. The fully dissolved sample was then shaped by EDM into a $5 \mathrm{x} 5 \mathrm{x}$ $10 \mathrm{~mm}$ parallelepiped with the hot-pressing direction along the long axis. The porosity was calculated with mass and volume measurements, using $6.45 \mathrm{~g} / \mathrm{cm}^{3}$ as the density of bulk NiTi [18].

Samples of the NiTi-steel composite were prepared for optical and SEM microscopy by mounting, grinding with 320 and 800 grit, and finally polishing with $9 \mu \mathrm{m}$ diamond suspension and $0.05 \mu \mathrm{m}$ colloidal silica suspension. Scanning Electron Microscopy (Hitachi SEM S-3400 II) was used to examine the microstructure and bonded powder boundaries, as well as to characterize the pore size and distribution. The composition of phases was determined by Energy Dispersive X-Ray Spectroscopy (EDS). Differential Scanning Calorimetry (DSC) was used to characterize the phase transformations under nitrogen cover gas with a heating/cooling rate of $5^{\circ} \mathrm{C} /$ minute on $30 \mathrm{mg}$ samples cut using a diamond saw. Samples were subjected to three consecutive cycles ranging from $-50^{\circ} \mathrm{C}$ to $100^{\circ} \mathrm{C}$. The transformation enthalpies were measured 
as the area under the DSC peaks, while martensite -start and -finish $\left(\mathrm{M}_{\mathrm{s}}\right.$ and $\left.\mathrm{M}_{\mathrm{f}}\right)$ and austenite start and-finish $\left(\mathrm{A}_{\mathrm{s}}\right.$ and $\left.\mathrm{A}_{\mathrm{f}}\right)$ were determined as the intersection of the tangents of inflection points and the baseline.

The $5 \times 5 \times 10 \mathrm{~mm}$ sample was characterized in uniaxial compression by cyclic loading to failure as described in [17]. The sample was heat-treated before each compression cycle to ensure a fully martensitic microstructure by heating to $100^{\circ} \mathrm{C}$ in boiling water for 5 minutes, cooling to $-196^{\circ} \mathrm{C}$ in a liquid nitrogen bath for 5 minutes, and then allowing the sample to return to room temperature. Compression testing was performed at a rate of $10^{-4} \mathrm{~s}^{-1}$ on a screw-driven load frame (Sintech 20/G) using an alignment cage to ensure parallelism. The surfaces of the sample were lightly ground with $600 \mu \mathrm{m}$ grit grinding paper to remove any oxide layers formed during the EDM process before mechanical testing. Strain was measured using crosshead displacement after compliance correction using the direct technique in [19]. Cyclic compression load-unload-recovery cycles were performed by loading the sample at ambient temperature in uniaxial compression to a maximum stress $\sigma_{\max }$ then unloaded, removed from the frame and thermally recovered as described above. The sample was subjected to successive load-unloadrecovery cycles, starting with $\sigma_{\max }=48 \mathrm{MPa}$ and increasing by $8 \mathrm{MPa}$, until failure of the sample was detected by a load drop.

\section{Results and Discussion}

\subsection{Formation of TiC}

At the hot-pressing temperature of $1020^{\circ} \mathrm{C}$, iron readily diffuses into the NiTi matrix unless prevented $[20,21]$ by the creation of a titanium carbide (TiC) layer at the wire surface. During electrochemical dissolution, the Fe-containing NiTi matrix near the steel wires dissolves 
together with the steel wire spaceholder [22]. However when steel with greater than 0.2 wt.\% carbon is used, a TiC layer forms between the wire spaceholder and the NiTi matrix during the high temperature densification step [14,17,23]. This layer prevents inter-diffusion between Fe and NiTi. Previously, low carbon steel meshes were used as a spaceholder, necessitating an additional carburizing processing step to ensure a carbon content high enough for $\mathrm{TiC}$ formation $[14,17,21,22]$. Here, commercially-available high carbon steel wires were used to eliminate the need for carburizing. The TiC layer was readily formed during hot pressing, labeled in Figure 2, with a thickness of $\sim 6 \mu \mathrm{m}$. This layer prevented iron diffusion into the NiTi powders, as confirmed with EDS, and the high carbon steel wires were well replicated as microchannels after electrochemical dissolution. A Ti-depleated layer of approximately the same thickness as the TiC layer formed between the TiC and NiTi matrix as Ti was depleted to form the TiC layer.

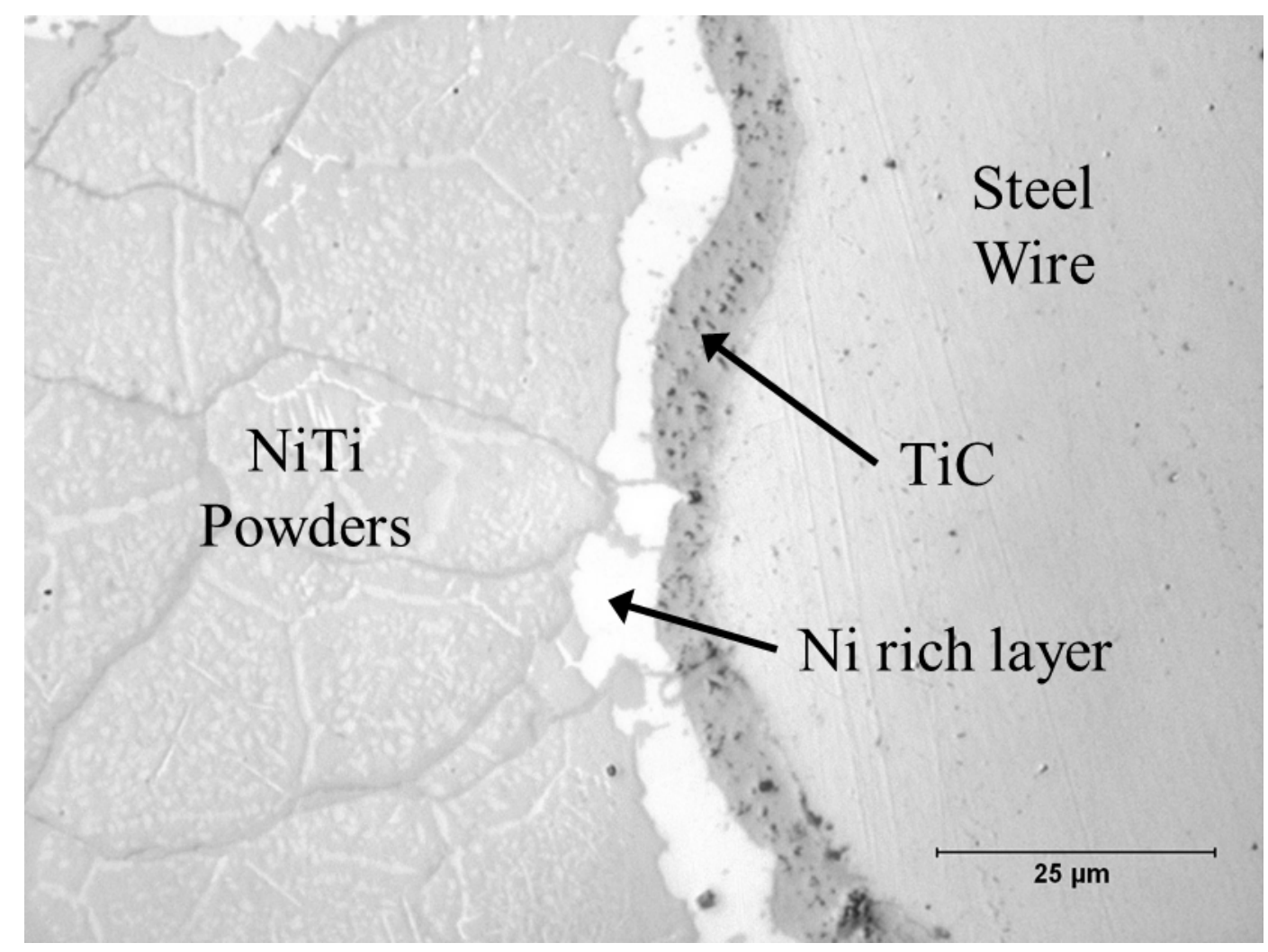


Figure 2: Optical micrograph of polished cross-section of NiTi-steel composite, showing TiC and Ti-depleated layers at the interface between the densified NiTi powders and a high-carbon steel wire spaceholder

\subsection{Macrostructure}

After the steel wire scaffold was electrochemically dissolved, orthogonally stacked microchannels within a continuous dense NiTi matrix are well replicated, as shown in Figure 3A. However rather than the original circular wire cross-section, these microchannels have ellipsoidal cross-sections. At the hot-pressing temperature, the steel wires softened and distorted under the $40 \mathrm{MPa}$ pressure used to densify of the powders, before the NiTi powders densified around the steel scaffold and the pressure became hydrostatic. The cross-sections displayed in Figure 3B have an aspect ratio of $0.74 \pm 0.03$, with a major $445 \pm 13 \mu \mathrm{m}$ axis perpendicular to the hot-pressing direction and a minor $330 \pm 12 \mu \mathrm{m}$ axis parallel (Figure 3B), averaged over 100 microchannel cross-sections using SEM. The wire cross-section before densification is outlined with yellow dashed lines. The $545 \pm 50 \mu \mathrm{m}$ edge-to-edge spacing between parallel channels, as measured from SEM images, remained similar to the nominal $500 \pm 50 \mu \mathrm{m}$ pre-densification wire spacing. The stacking grooves to space the steel wires in scaffold were $500 \mu \mathrm{m}$ to allow the $400 \mu \mathrm{m}$ diameter wires to easily slide into the groove. This clearance caused variance in the edge-to-edge spacing. 

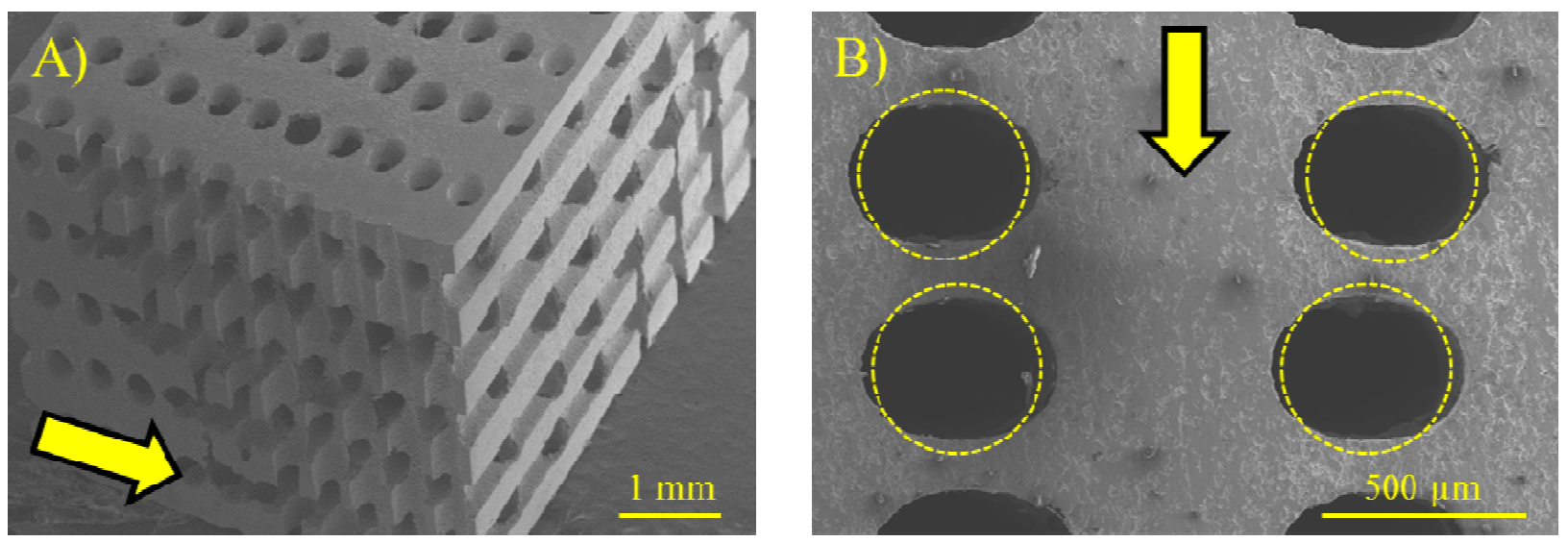

Figure 3: SEM micrographs of the EDM-cut surfaces of the 3D interconnected porous NiTi structure. (A) Overall view showing orthogonally intersecting microchannels; (B) detailed view showing ellipsoidal cross-sections. The hot-pressing axis is marked with a thick arrow and predensification wire cross-sections are indicated with dashed circles.

Prior to densification, the $400 \mu \mathrm{m}$ wire cross-section defined the spacing between stacked layers of wires. However the spacing between channels along the densification direction was measured as $130 \pm 12 \mu \mathrm{m}$. . As the NiTi-steel composite was densified, the tap-packed NiTi powders between layers of wires with the same orientation were distorted and packed together, while the wires were compressed. Both of these effects reduced the microchannel spacing between layers of microchannels with the same orientation along the densification direction. At the hot-pressing temperatures, the harder NiTi powders also indented the softer steel wires, creating a textured channel surface wall visible in Figure 4B.

The microchannels are connected along the densification direction by windows or fenestrations, as illustrated in Figure 4A. These windows were created by the contact of adjoining spaceholder wires layers, which are outlined with dashed lines in Figure 4A. This contact point was broadened during densification when the wires flattened, forming a larger 
window. The windows, measured by SEM to be $510 \pm 35 \mu \mathrm{m}$ in diameter, are aligned in the densification direction, and are continuous through the entire structure, as determined by visual inspection against a lighted background. Because the $44-63 \mu \mathrm{m}$ NiTi powders were unable to fully pack into the cusps at the wire contact areas during densification, the perimeters of the windows (at the intersection of the dashed microchannel outlines in Figure 4A) are more irregularly shaped than the smooth ellipsoidal microchannel cross-sections outlined in Figure 4B.
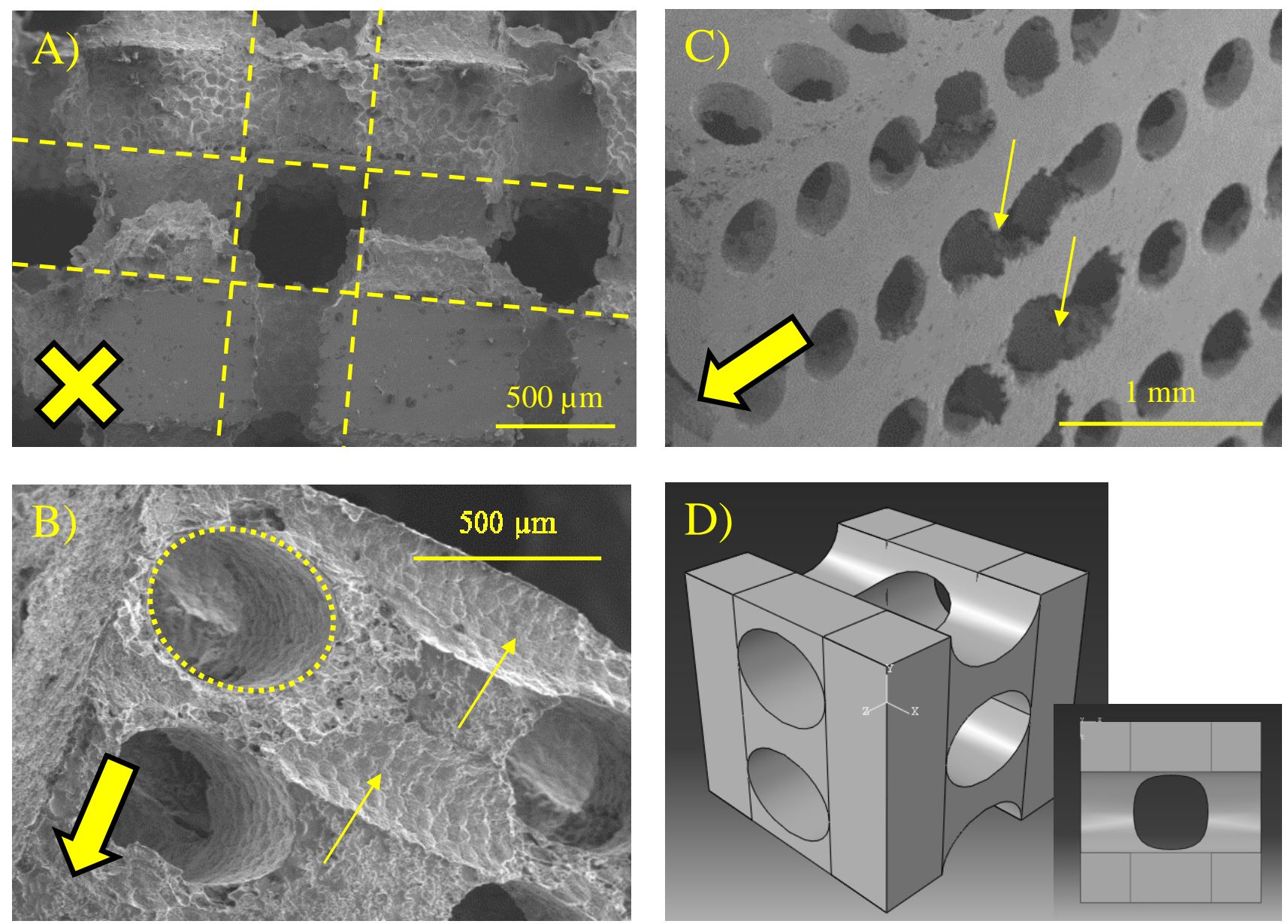

Figure 4: SEM micrographs of EDM-cut surfaces showing A) interconnection windows

(fenestrations) with in-plane microchannels outlined, B) textured microchannel walls (fine arrows) and a microchannel cross section outlined in dashed yellow, and C) missing NiTi bridges 
within the structure (fine arrows). D) The structure is schematically displayed as columns and bridges, with the interconnection window in the inset (same view as A)). The thick yellow arrows indicate the hot pressing direction, where the $\mathrm{X}$ indicates that the direction is into the page.

Alternatively, the porous NiTi structure can be described as columns parallel to the densification direction connected by struts perpendicular to the densification direction, as schematically displayed in Figure 4D. The fenestrations, forming channels parallel to the densification direction, separate the columns, while the microchannels created by the steel wires surround the struts. In a few locations on the machined surface, struts are missing and channels are connected by voids. One such location is shown in Figure 4C. These indicate regions which were not fully filled by NiTi powders prior to densification, which were shielded by surrounding steel wires. These voids are undesirable, as they create local stress concentrations and reduced load-bearing regions during mechanical testing, which may initiate cracking and structure failure. They could be minimized in future work by using finer NiTi powders, or vacuum infiltration of the scaffold with a NiTi-water slurry.

The porosity of the structure was measured as $61.5 \pm 0.3 \%$. Prior to densification, the steel scaffold in the NiTi-steel composite comprised $34 \%$ of the volume within the hot-pressing die. Assuming dense packing for mono-sized spheres, $70 \%$ of the volume surrounding the scaffold would be filled with NiTi powder. Dividing the volume of the steel scaffold (calculated by mass conversion) by the combined volume of the scaffold and this theoretical volume of NiTi powders (the volume of the densified steel-NiTi composite), gives a channel volume fraction of $57.6 \%$ for perfectly packed mono-sized spheres, slightly underestimating the measured porosity 
of the structure. Factors that can affect the porosity might be less than optimal packing of the powders due to the confinement effect of the wires, the powder size distribution, and the presence of occasional unfilled regions between wires, such as in Figure 4C.

\subsection{Microstructure}

The fully densified NiTi matrix, prior to dissolution of the steel wire spaceholder, is shown in a SEM micrograph in Figure 5A. Prior NiTi powders are visible between two steel wires, with their shapes distorted from their original spherical shape by the densification. A few small voids are visible. The self-accommodated martensite microstructure is also observable in Figure 5B within the NiTi powder particles, as well as a few micron-size gray precipitates (as indicated by an arrow in Figure 5B), which are identified with EDS as $\mathrm{Ti}_{2} \mathrm{Ni}$.
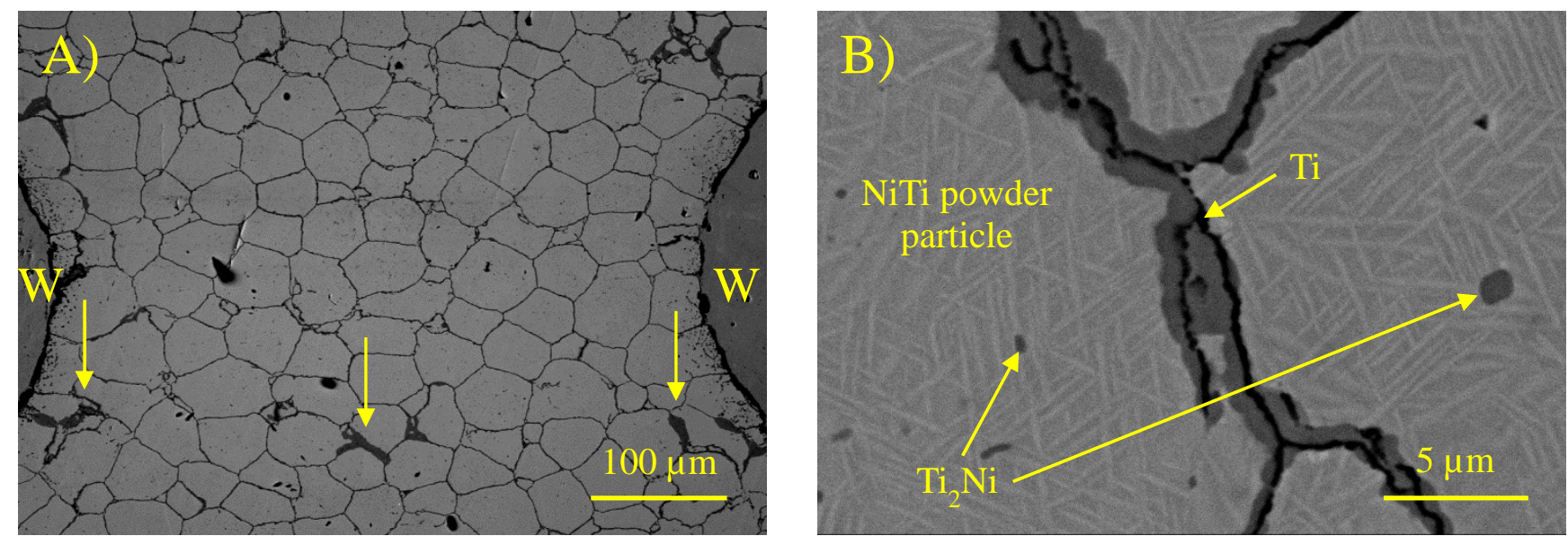

Figure 5: SEM micrographs of cross-sections of the densified NiTi-steel composite displaying A)

NiTi matrix between two steel wires (labeled W) with iron contaminated regions marked with thin arrows, and B) black TiC layers at prior NiTi powder boundary surrounded by dark gray titanium-depleted $\mathrm{NiTi}$ zone, with $\mathrm{Ti}_{2} \mathrm{Ni}$ particles in grey NiTi powder matrix. 
The steel wires are covered with a thin black phase as expected from Figure 2, identified through EDS as TiC. Unexpectedly, the prior NiTi powder boundaries are fully decorated by TiC (as determined by EDS). The carbon most probably originates from the boron nitride lubricant used to prevent bonding of the sample to the TZM die during hot pressing, which contains $2 \%$ carbon as a binder according to the manufacturer. During wet packing, it is likely that the binder was dissolved and redeposited on the surface of the NiTi powders, where its presence later formed $\mathrm{TiC}$.

At most powder boundaries, this black-shaded TiC phase is approximately $0.3 \mu \mathrm{m}$ thick, though, as with the NiTi-steel TiC interface, it varies in thickness depending on the angle of the cross-section. The TiC interface is surrounded by a gray titanium-depleted NiTi zone, caused by the formation of TiC. The thickness of the layer has high variation, but is approximately $1.4 \pm$ $0.6 \mu \mathrm{m}$. Both the TiC and Ti-depleated layers are present in small amounts at nearly all of the powder boundaries, indicating global contamination. In several locations, such as the ones indicated with arrows in Figure 5A, there are larger regions of titanium depletion which also contain $1.5-3$ at $\% \mathrm{Fe}$. The origin of this Fe contamination is unclear, but it is possible that during the wet packing procedure, small particles of rust formed on the wire scaffold and were dispersed through the NiTi powder prior to hot pressing. These regions are more common closer to the wire scaffold interface, and are approximately $5.6 \mu \mathrm{m}$ thick surrounding the TiC layer.

Transformation temperatures and enthalpies for the 3D interconnected porous structure and the prealloyed NiTi powder are listed in Table 1. The phase transformation was single-step during both heating and cooling, displaying only austenite and martensite phases. The enthalpy is lower for the porous structure as compared to NiTi powder for both heating and cooling 
transformations. The $\mathrm{A}_{\mathrm{S}}$ and $\mathrm{M}_{\mathrm{F}}$ temperatures are unchanged between powder and porous structure form, but both $\mathrm{A}_{\mathrm{F}}$ and $\mathrm{M}_{\mathrm{S}}$ decrease by approximately $20^{\circ} \mathrm{C}$.

\begin{tabular}{|c|c|c|c|c|c|c|c|}
\hline \multirow[t]{2}{*}{ Specimen } & \multicolumn{2}{|c|}{ Enthalpy $\left(\mathrm{J} \mathrm{g}^{-1}\right)$} & \multicolumn{4}{|c|}{ Transformation Temperatures $\left({ }^{\circ} \mathrm{C}\right)$} & \multirow[t]{2}{*}{ Porosity $(\%)$} \\
\hline & Heating & Cooling & $A_{S}$ & $A_{F}$ & $\mathrm{M}_{\mathrm{s}}$ & $\mathrm{M}_{\mathrm{F}}$ & \\
\hline NiTi Powder ${ }^{1}$ & 24 & 23 & 54 & 90 & 61 & 28 & $=$ \\
\hline Porous Structure & 15 & 17 & 53 & 74 & 42 & 26 & 60 \\
\hline
\end{tabular}

Table 1: Enthalpies and phase transformation temperatures of prealloyed NiTi powders and the 3D interconnected porosity NiTi structure. ${ }^{1}[14]$

The decrease in enthalpy is similar to that observed in HIPed NiTi structures with 2-D interconnected wavy microchannels [17], as well as a similar shift in the transformation temperatures. Porosity does not affect transformation behavior [6,7]. However, the formation of TiC creates a corresponding gradient of Ti depletion at the surface of pores. The nontransforming $\mathrm{TiC}$, at both the microchannel walls and powder boundaries decreases the volume of transforming material, decreasing enthalpy. NiTi regions with high Ti depletion will not transform, further decreasing the enthalpy. Where Ti-depleated (Ni-rich) NiTi transforms, it will be at lower transformation temperatures [24], broadening the DSC transformation peaks and shifting them to lower temperatures. NiTi further from the powder boundary would be expected to transform similarly to loose NiTi powders. However the convolution of the NiTi of the prealloyed composition and the Ti depletion regions stretch the phase transformation towards lower temperatures.

\subsection{Mechanical properties}


The stress-strain curve under monotonic compressive loading to critical failure at $\sim 1 \%$ strain is shown in Figure 6. The sample displayed linear elastic behavior until a load drop at $0.64 \%$ strain, indicating initiation of failure. The sample continued to accommodate load until critical failure. Cyclic loading cycles all fall directly on this curve, with no transformation plateau, and no residual strains measured upon unloading after each cycle. Experimental data below $35 \mathrm{MPa}$ were convoluted with compliance due to alignment of the cage and sample, so the curve was extrapolated to zero stress assuming a linear elastic response. Since orientation of martensite can initiate even at low stresses, especially in porous samples, the linear response is not a true Young's modulus, but an effective stiffness. The sample deformed at low stresses with an effective stiffness of $\mathrm{E}=14 \mathrm{GPa}$ until a load drop indicated non-catastrophic fracture at $88 \mathrm{MPa}$ and $0.64 \%$ strain. It continued to accommodate load until critical failure at $90 \mathrm{MPa}$ and $~ 1 \%$ strain. Brittle fracture occurred at the critical failure, extending cracks entirely through the upper layer of microchannels in contact with the upper loading platen, perpendicular to loading. After critical failure, the cracking sheared the upper layer of microchannels completely from the bulk material, preventing further loading and densification of the structure. 


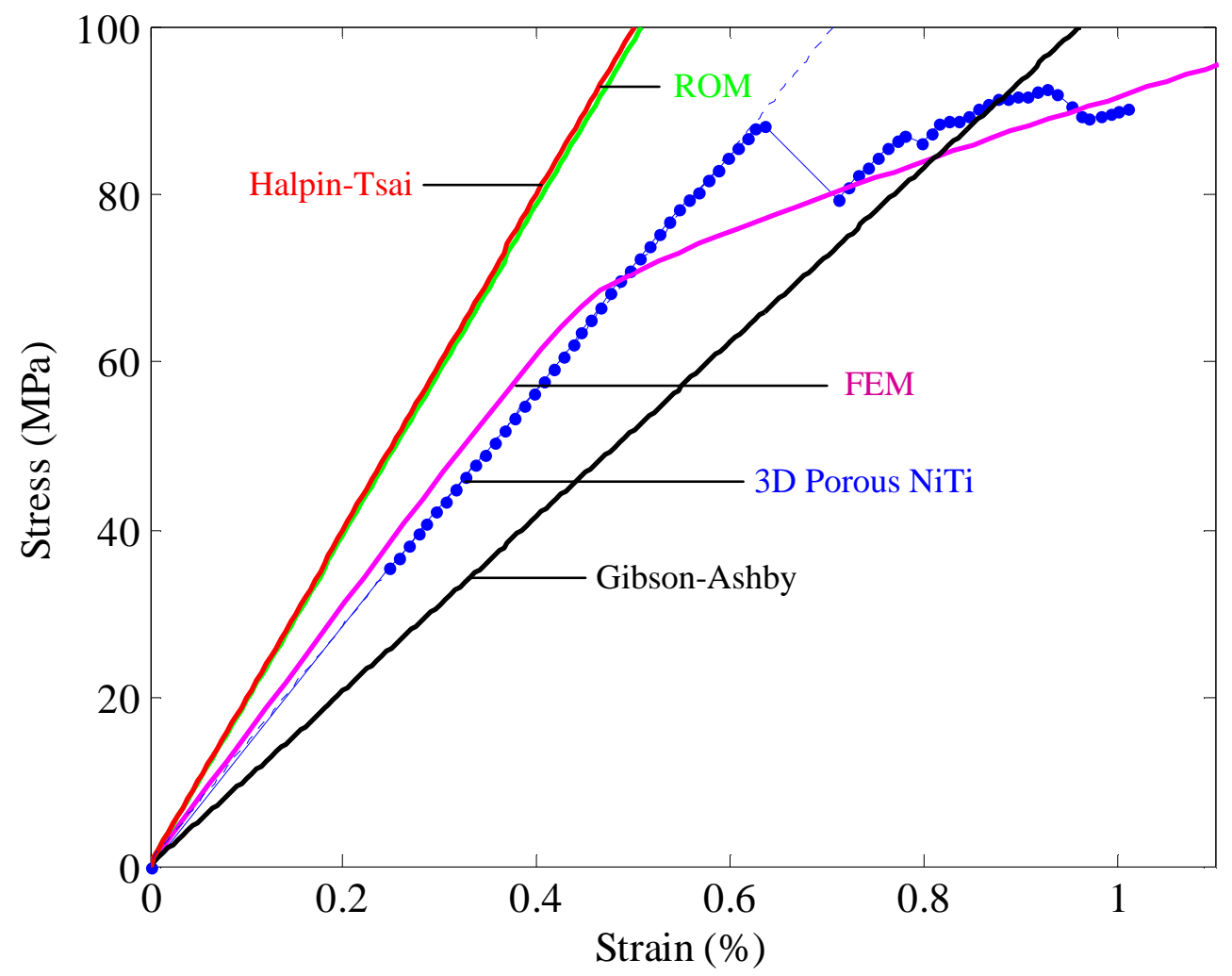

Figure 6: Experimental compressive stress-strain curves under monotonic loading to failure with a dashed linear fit line, along with Gibson-Ashby, ROM, and Halpin-Tsai elastic models, and FEM predictions.

The fracture surface, exposed by the sheared-off layer of microchannels previously in contact with the top loading platen is shown in Figure 7. Two cracks, indicated with thin arrows, follow the NiTi powder boundaries along the TiC interface as expected from its brittleness. The fractured cross-section of a column is outlined with a yellow square, where exposed prior powder boundaries are clearly visible. Cracking initiated within columns propagated transversely to the applied load, while cracking within bridges propagated parallel to the applied load, fracturing the structure. The self-accommodated microstructure visible within the NiTi powders (Figure 5B), indicates that the structure should deform through martensite variant 
orientation. However, the continuous network of stiffer, yet brittle [25], TiC phase constrains the powders. The TiC phase fractured before martensite orientation of the NiTi powders was apparent on a macroscopic scale, and the structure failed through cracking along the powder boundaries.

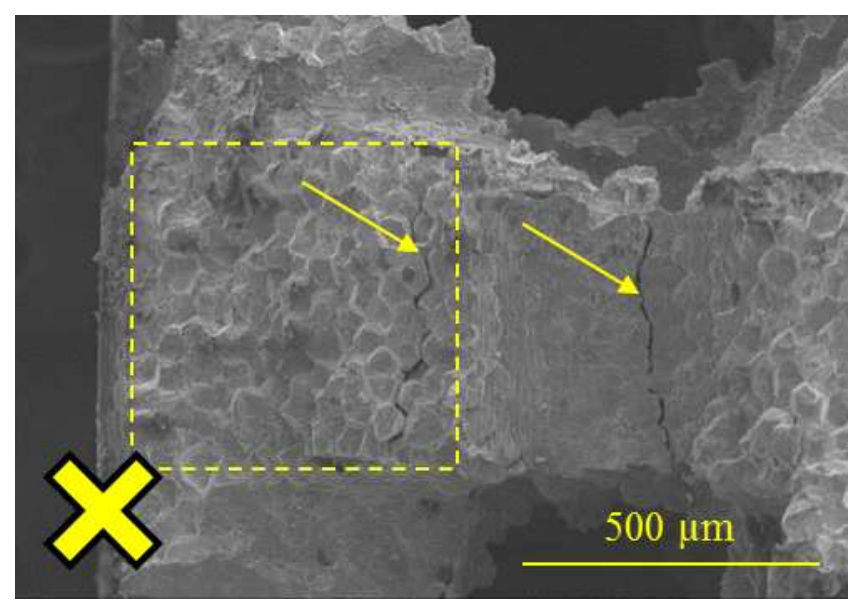

Figure 7: SEM micrograph of the fracture surface of the 3D interconnected porous NiTi structure.

Powder boundaries are exposed through the cross-section of the outlined column, and cracks indicated with thin arrows. The loading axis is into the image.

\section{Modeling of Stiffness}

\subsection{Gibson-Ashby Foam Model}

The Gibson-Ashby equations correlate the stiffness and strength of a cellular structure with porosity [26], and are used here as a first-order approximation for the stiffness of this 3D porous structure. The model is based on strut bending of an isotropic cellular structure where the Young's modulus $\mathrm{E}$ of the cellular structure is related to that of the bulk material $\mathrm{E}_{\mathrm{M}}$ by:

$$
E=E_{M} C\left(\frac{\rho}{\rho_{M}}\right)^{2}
$$


where $\mathrm{C}$ accounts for geometric constants of proportionality, and $\rho$ and $\rho_{M}$ are the densities of the cellular structure and bulk material respectively. The constant $\mathrm{C}$ has been shown to be unity [26] for solid struts, though could be reduced with porosity within the strut matrix [27]. The porosity, $p$, of the structure is related to the ratio of densities by $1-p=\rho / \rho_{M}$.

The stiffness of the 3D interconnected porous structure calculated using the GibsonAshby model is plotted in Figure 6 using $C=1, E_{M}=65 \mathrm{GPa}$, the stiffness of monolithic HIPed NiTi [17], and a structure porosity of $60 \%$. The resulting Young's Modulus, E = 10.4 GPa, underestimates the experimental stiffness by $\sim 4 \mathrm{GPa}$. The Gibson-Ashby cellular structure is assembled with offset cells, such that the vertical members meet at the midpoints of the horizontal members, resulting in bending loads. However in the case of our 3D interconnected porous structure, the primary loading mechanism is compression of aligned columns. Considering the differences in deformation mechanisms between the two geometries, the Gibson-Ashby model gives a surprisingly close estimate for the stiffness.

\subsection{Continuous Fiber Models}

A more accurate estimate may be to consider the geometry of the structure as either (1) aligned columns with connecting struts, or (2) aligned (orthogonally stacked) microchannels. In these cases, continuous fiber models can be applied which estimate the stiffness of a composite material of NiTi and a porous phase with zero stiffness. The structure stiffness can be estimated using the rule-of-mixtures (ROM) Voigt bound (parallel model) for (1) where the NiTi columns are taken as aligned fibers, with a "matrix" of pores between them having zero stiffness. In this case the connecting struts are assumed to carry no load, and are treated as a component (thus volume fraction) of the porous phase. The Reuss bound (series model) for ROM cannot use a 
zero stiffness for the pore moduli, and instead we use the semi empirical Halpin-Tsai relation for (2), the transversely loaded aligned fibers, where the fibers are zero stiffness microchannels within a NiTi matrix.

The Young's modulus for the 3D interconnected porous "composite" structure can be estimated through ROM as:

$$
E_{R O M}=f E_{f}+(1-f) E_{M}
$$

where $f$ is the volume fraction of the NiTi column fibers, and $\mathrm{E}_{f}$ is the bulk Young's Modulus for HIPed NiTi from above. $\mathrm{E}_{M}$, the stiffness of the pore matrix, is set to 0 . The $3 \mathrm{D}$ interconnected porous structure is highly regular, and a schematic of the representative volume unit is shown in Figure 8 illustrating the columns and struts. The overall structure is $40 \%$ dense NiTi. The columns are defined by the spacing between the major axis of the pores, with a crosssection of $545 \times 545 \mu \mathrm{m}$. The structure can therefore be partitioned into 30.3 vol.\% columns, 9.7 vol.\% struts, and 60 vol.\% porosity. Using these values, the ROM estimation for the Young's modulus is $\mathrm{E}_{\mathrm{ROM}}=19.7 \mathrm{GPa}$, which is plotted along with the experimental and other model results in Figure 6.

The semi-emperical Halpin-Tsai model estimates the stiffness of transversely loaded aligned fibers. In this case, the microchannels are treated as the aligned fibers with zero stiffness, and both the columns and bridges are considered for the NiTi matrix. The Young's modulus is then determined by:

$$
E_{H T}=\frac{E_{M}\left(1+\xi \eta V_{f}\right)}{1-\eta V_{f}} \quad \text { where } \quad \eta=\frac{E_{f}-E_{M}}{E_{f}+\xi E_{M}}
$$

Because the fiber stiffness $\mathrm{E}_{f}=0$, the equation reduces such that $\eta=-1 / \xi$. $\mathrm{E}_{\mathrm{M}}$ is bulk stiffness of NiTi, while $\mathrm{V}_{f}$ is the volume fraction of fibers, or the porosity of the structure. The parameter $\xi$ is a curve fitting parameter that experimentally falls between $1<\xi<2$. However for square 
arrays $\xi=2$ is commonly assumed [28] which we use here rather than fitting $\xi$ to our data. The Halpin-Tsai model then estimates the stiffness to be $\mathrm{E}_{\mathrm{HT}}=20.0 \mathrm{GPa}$, also plotted on Figure 6 .

The two continuously aligned fiber models yield very similar results, and overestimate experimental stiffness by $\sim 6 \mathrm{GPa}$. The Halpin-Tsai estimation is slightly higher than the ROM estimation. While the Haplin-Tsai model is typically the upper bound of the two models, it is a semi-empirical model and adjustment of the fitting parameter $\xi$ makes it not an absolute upper bound, making the difference between the two estimations not significant. The geometric models are very similar to the 3D interconnected porous structure, with similar deformation modes. However, the models are purely linear elastic. NiTi also deforms through orientation of martensitic variants, even at low loads. In addition, the geometry of the microchannels introduces local stress concentrations, enabling martensitic orientation to initiate at lower average sample strains. Both of these phenomena will lower the apparent stiffness of the structure.

\subsection{Stebner-Brinson Finite Element Model}

Finite-element modeling using the Stebner-Brinson SMA model incorporates the actual geometry of the porous structure and includes effects such as martensitic variant orientation [29]. Porous NiTi materials are typically difficult to model, as irregularly spaced and shaped porosity requires large representative volumes with many elements, making them computationally expensive. Because the 3D interconnected porous geometry developed here is highly regular, a representative unit cell can be used with a relatively low number of elements. Choosing a representative unit cell with 2 orthogonally stacked microchannels, the 3D model was optimized with 63,000 tetragonal elements. Mechanical properties for the HIPed NiTi 
matrix material in the model were calibrated to the stress-strain response of HIPed NiTi in literature [30], and phase transformation temperatures were taken from DSC values above.

The global stress-strain response from FEM is plotted in Figure 6. Macroscopically, deformation is linearly elastic and the stiffness of the structure is $15.4 \mathrm{GPa}$. This value is in good agreement with experimental results, with only a small overestimation of $1.2 \mathrm{GPa}$.

However, the stress-strain curve is the average structure response, due to the macroscopic geometry. Locally, strains are higher, and these high-strain regions undergo martensitic orientation at lower applied stresses without detection in the average response, or without a change in slope in the nominally elastic region of the stress-strain curve.

The average stress-strain curve is shown in Figure 8, together with representative unit cells showing, at various macroscopic strains, localized strains $\varepsilon$ and the volume fraction of oriented martensite $\xi$. Self-accommodated martensite is displayed in gray, and increasing volume fractions of oriented martensite as shown in color. At $0.3 \%$ average macroscopic strain, the maximum localized strain is $0.75 \%$. The strain concentration is localized at the apex of the major axis of the elliptical channels, or the point at which the loaded columns connect to the porous microchannels. The bridges are completely unloaded, with $0 \%$ strain, and the average strain of the columns is $\sim 0.3 \%$ strain, or the same as the average strain response. The strain in the columns is all linearly elastic strain, as the volume fraction of oriented martensite is $0 \%$. However at the localized strain concentration, $9 \%$ of the volume of the martensite in the affected (colored) region has oriented. 


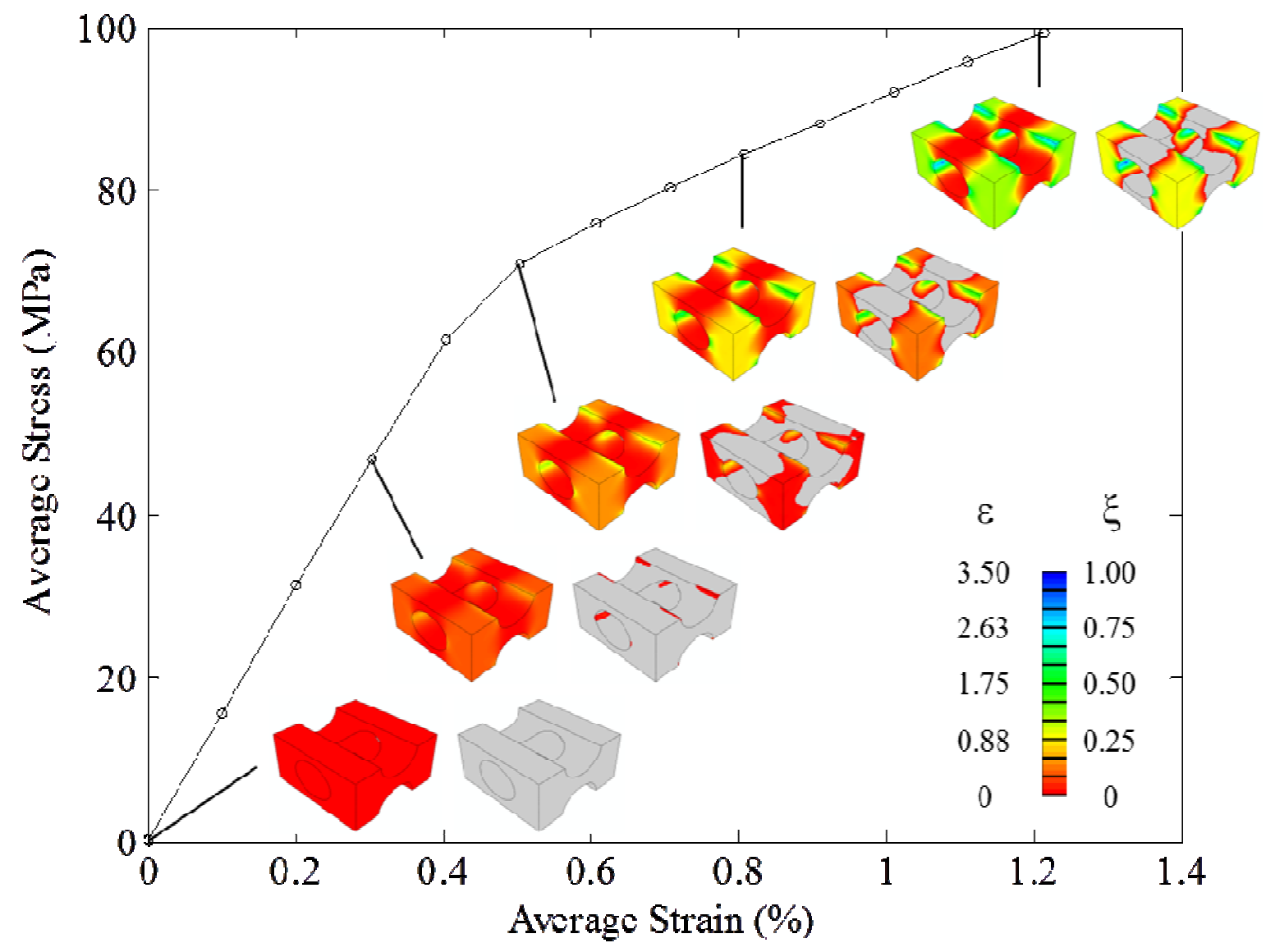

Figure 8: Average macroscopic stress-strain FEM curve with representative unit cell showing calculated localized strain $\varepsilon$ (left) and volume fraction of oriented martensite $\xi$ (right). Gray regions indicate self-accommodated martensite.

With increasing applied load, the strain in the columns remains the same as the macroscopic average strain response. At $0.5 \%$ average strain (or column strain), just above the onset of the martensite orientation plateau at $0.45 \%$ average strain, the maximum localized strain has increased to $1.4 \%$, and the self-accommodated martensite in the columns has begun to orient. These region forms bands within the columns connecting the localized strain concentrations, bowing away from the bridges. With increasing applied load, this region extends towards the 
bridges, and by $1.0 \%$ average strain the entire column has initiated orientation, with $\sim 25$ vol\% of the martensite in the columns oriented. At the maximum strain calculated in the model, $1.2 \%$ average strain, $\sim 30$ vol\% of the martensite in the columns is oriented. The maximum localized strain continues to increase at a rate of three times the average strain, and by $1.2 \%$ average strain the maximum localized strain is $3.5 \%$, with $85 \%$ local volume fraction of oriented martensite.

The elastic response between the model and experimental results are very similar. The average response experimentally is limited by the brittle TiC interface between NiTi powder particles, preventing an average orientation plateau. As with the strain-concentrated region, it is possible that transformation is occurring within NiTi powders, especially near the apex of the major axis of the elliptical channels. However, before this could be detected in the average stress-strain response, cracking and fracture initiated along the TiC interface.

\section{Conclusions}

NiTi porous structures with fully 3D interconnected microchannels were fabricated by hot-pressing a preform of prealloyed NiTi powders containing a high-carbon steel wire scaffold. The scaffold was then electrochemically dissolved, resulting in a replicated, regular 3D network of microchannels, orthogonally interconnected in 3d, with ellipsoidal cross-sections and a volume fraction of $60 \%$. Using high-carbon steel wires formed a thin TiC layer at the NiTi-steel wire interface during densification without requiring additional carburizing, and prevented iron from diffusing into the NiTi matrix. Thus the microchannels replicate with high fidelity the steel scaffold after electrochemical dissolution. Carbon binder contamination from the die lubricant also formed $\mathrm{TiC}$ at all NiTi powder boundaries, embrittling the structure which cracked along the 
TiC interface at a relatively low stress of $88 \mathrm{MPa}$, insufficient to generate plasticity by martensite orientation. This can be avoided by refined processing to prevent $\mathrm{C}$ contamination.

The measured elastic stiffness of $14 \mathrm{GPa}$ was compared to porous and composite models, as well as finite element modeling. Each of the models estimated the stiffness well despite varying geometry and deformation model assumptions. The structure can be partitioned into continuous columns, loaded in parallel, with unloaded connecting bridges. This was supported with finite element modeling where the average strain in the columns matched the average structure strain, with unloaded bridges. Modeling indicated that strain concentrations were localized at microchannel walls perpendicular to the applied load, and were approximately three times greater than the average stain. Martensite orientation initiated at these strain concentrations, then propagated through the loaded columns. The brittle TiC layer constrained deformation of the NiTi powders, leading to structure failure before orientation was observable in the average structure response. However, it is likely that local regions of self-accommodated martensite within NiTi powders oriented, especially near the strain concentrations. With prevention of contamination eliminating the TiC layer between NiTi powders, finite element modeling indicates that the structure would have excellent shape memory properties as well.

The fabrication method presented here allows for a highly controlled geometry of microchannels, coupled with a low effective stiffness. The scalability of the method is limited by the loading capacity of the hot pressing equipment. The volume fraction, orientation, shape, and spatial distribution of the microchannels are all fully controlled with this method. Fully 3D interconnected microchannels increase the permeation of the structure and enable efficient fluid transport. This makes the structure attractive for biomedical applications, specifically bone implants where increased nutrient and waste transport allow bone cells to grow and propagate 
deep within the structure. The potential shape-memory properties achievable through optimized processing would also make the structure effective for energy absorption or actuators.

\section{Acknowledgements}

The authors would like to thank P. Zhu for her assistance with the finite element modeling. This research was supported by the Office of Army Research (W911NF-12-10013/P00002), the National Science Foundation (CMS-0404291), as well as Department of Energy (DE-SC0010594). C. Bewerse also gratefully acknowledges a National Defense Science and Engineering Graduate (NDSEG) Fellowship, 32 CFR 168a, awarded by the Air Force Office of Scientific Research (DoD). This work made use of the EPIC facility (NUANCE CenterNorthwestern University), which has received support from the MRSEC program (NSF DMR1121262) at the Materials Research Center, and the Nanoscale Science and Engineering Center (EEC-0118025/003), both programs of the National Science Foundation; the State of Illinois; and Northwestern University.

\section{References}

[1] K.L. Fukami-Ushiro, D. Mari, D.C. Dunand, Metall. Mater. Trans. A 27A (1996) 183.

[2] K. Otsuka, C. Wayman, Shape Memory Materials, Cambridge University Press, Cambridge, UK, 1998.

[3] A. Bansiddhi, T.D. Sargeant, S.I. Stupp, D.C. Dunand, Acta Biomater. 4 (2008) 773.

[4] G. Ryan, A. Pandit, D.P. Apatsidis, Biomaterials 27 (2006) 2651.

[5] M.H. Elahinia, M. Hashemi, M. Tabesh, S.B. Bhaduri, Prog. Mater. Sci. 57 (2011) 911.

[6] a Bansiddhi, D. Dunand, Intermetallics 15 (2007) 1612. 
[7] a Bansiddhi, D.C. Dunand, Acta Biomater. 4 (2008) 1996.

[8] A. Bansiddhi, D.C. Dunand, J. Mater. Res. 24 (2009) 2107.

[9] T. Aydoğmuş, Ş. Bor, J. Alloys Compd. 478 (2009) 705.

[10] S.M. Oppenheimer, D.C. Dunand, Mater. Sci. Eng. A 523 (2009) 70.

[11] B.Y. Li, L.J. Rong, Y.Y. Li, V.E. Gjunter, Acta Metall. 48 (2000) 3895.

[12] P. Novák, L. Mejzlíková, A. Michalcová, J. Čapek, P. Beran, D. Vojtěch, Intermetallics 42 (2013) 85.

[13] M. Sugiyama, A.-K. Hyun, M. Tane, H. Nakajima, High Temp. Mater. Process. 26 (2007) 297.

[14] A.J. Neurohr, D.C. Dunand, Acta Biomater. 7 (2011) 1862.

[15] C. Bewerse, L.C. Brinson, D.C. Dunand, J. Mater. Process. Technol. 214 (2014) 1895.

[16] I. ASTM, Annu. B. ASTM Stand. (2008) 1.

[17] A.J. Neurohr, D.C. Dunand, Acta Mater. 59 (2011) 4616.

[18] C.M. Jackson, H.J. Wagner, R.J. Wasilewski, 55-Nitinol-The Alloy with a Memory: Its Physical Metallurgy, Properties, and Applications, Washington, DC, 1972.

[19] S.R. Kalidindi, a. Abusafieh, E. El-Danaf, Exp. Mech. 37 (1997) 210.

[20] P.J. Kwok, S.M. Oppenheimer, D.C. Dunand, Adv. Eng. Mater. 10 (2008) 820.

[21] D.J. Jorgensen, D.C. Dunand, Mater. Sci. Eng. A 527 (2010) 849.

[22] D.J. Jorgensen, D.C. Dunand, Acta Mater. 59 (2011) 640.

[23] T. Momono, T. Enjo, K. Ikeuchi, ISIJ Int. 30 (1990) 978.

[24] J. Frenzel, E.P. George, a. Dlouhy, C. Somsen, M.F.-X. Wagner, G. Eggeler, Acta Mater. 58 (2010) 3444.

[25] R. Vaidyanathan, M.A.M. Bourke, D.C. Dunand, Acta Mater. 47 (1999) 3353.

[26] L.J. Gibson, M.F. Ashby, Cellular Solids: Structure and Properties, 2nd ed., Cambridge University Press, Cambridge, United Kingdom, 1997.

[27] H. Hagiwara, D.J. Green, J. Am. Ceram. Soc. 70 (1987) 811. 
[28] I.M. Daniel, O. Ishai, in:, Eng. Mech. Compos. Mater., 2nd ed., Oxford University Press, New York, NY, 2006, pp. 51-56.

[29] a. P. Stebner, L.C. Brinson, Comput. Methods Appl. Mech. Eng. (2013).

[30] K.. Fukami-Ushiro, D.C. Dunand, Metall. Mater. Trans. A 27A (1996) 193.

\section{Figure Captions}

Figure 1: Assembled and sintered spaceholder scaffold constructed with orthogonally stacked high-carbon steel wires with a diameter of $400 \mu \mathrm{m}$.

Figure 2: Optical micrograph of polished cross-section of NiTi-steel composite, showing TiC and Ti-depleated layers at the interface between the densified NiTi powders and a high-carbon steel wire spaceholder

Figure 3: SEM micrographs of the EDM-cut surfaces of the 3D interconnected porous NiTi structure. (A) Overall view showing orthogonally intersecting microchannels; (B) detailed view showing ellipsoidal cross-sections. The hot-pressing axis is marked with a thick arrow and predensification wire cross-sections are indicated with dashed circles.

Figure 4: SEM micrographs of EDM-cut surfaces showing A) interconnection windows (fenestrations) with in-plane microchannels outlined, B) textured microchannel walls (fine arrows) and a microchannel cross section outlined in dashed yellow, and C) missing NiTi bridges within the structure (fine arrows). D) The structure is schematically displayed as columns and bridges, with the interconnection window in the inset (same view as A)). The thick yellow 
arrows indicate the hot pressing direction, where the $\mathrm{X}$ indicates that the direction is into the page.

Figure 5: SEM micrographs of cross-sections of the densified NiTi-steel composite displaying A) NiTi matrix between two steel wires (labeled W) with iron contaminated regions marked with thin arrows, and B) black TiC layers at prior NiTi powder boundary surrounded by dark gray titanium-depleted NiTi zone, with $\mathrm{Ti}_{2} \mathrm{Ni}$ particles in grey NiTi powder matrix.

Figure 6: Experimental compressive stress-strain curves under monotonic loading to failure with a dashed linear fit line, along with Gibson-Ashby, ROM, and Halpin-Tsai elastic models, and FEM predictions.

Figure 7: SEM micrograph of the fracture surface of the 3D interconnected porous NiTi structure. Powder boundaries are exposed through the cross-section of the outlined column, and cracks indicated with thin arrows. The loading axis is into the image.

Figure 8: Average macroscopic stress-strain FEM curve with representative unit cell showing calculated localized strain $\varepsilon$ (left) and volume fraction of oriented martensite $\xi$ (right). Gray regions indicate self-accommodated martensite.

Table 1: Enthalpies and phase transformation temperatures of prealloyed NiTi powders and the 3D interconnected porosity NiTi structure. ${ }^{1}[14]$ 